\title{
A double-blind placebo-controlled heart rate variability investigation to evaluate the quantitative effects of the organic nanoscale aeon patch on the autonomic nervous system
}

\author{
Homer Nazeran \\ Department of Electrical and Computer Engineering and Biomedical Engineering Graduate Programs University of Texas at El \\ Paso, USA
}

\begin{abstract}
In this first double-blind-placebo-controlled investigation of the Aeon Patch, electrocardiographic data were acquired from 50 well-hydrated volunteers [21 females and 29 males, age: 19-79, weight: 117 - 334 lbs, height: 5'-6',3"], under 3 different conditions for a total duration of $15 \mathrm{~min}$ (5 min/condition). Condition1: Control (C) - while wearing no Patch, Condition 2: Placebo (P) or Active (A) - after wearing either the Placebo or the Aeon Patch for $20 \mathrm{~min}$, and Condition 3: Active (A) or Placebo (P) - after wearing either the Aeon or the Placebo Patch for $20 \mathrm{~min}$. There was a washout period of 15 min between Conditions 2 and 3. All involved in the investigation were blind to the (A) and (P) Patches as they looked exactly the same and were assigned in a random fashion. The hypothesis to be tested was: Wearing the Aeon Patch for 20 min reduces stress. Data were first quality assured and those subjects who showed a parasympathetic response after wearing the Patch within $20 \mathrm{~min}$ were identified as early responders. Thirty subjects (60\%) achieved a relaxed state after wearing the Aeon Patch as early as 20 min. Statistical analysis (one-sample inference) was used to compare the spectral features of the responders. The normalized LF/HF decreased significantly $(24 \% \pm 9 \%$ after $20 \mathrm{~min})$ in condition (A) compared to condition $(\mathrm{P})$ with a $p$-value $<0.047(\mathrm{n}=30)$ in responders. Therefore, the hypothesis that wearing the Aeon Patch for 20 min reduces stress was accepted as true.
\end{abstract}

Keywords acupuncture, aeon nanoscale organic patch, heart rate variability signal analysis, lifewave, stress reduction, CV6 acupuncture point

\section{INTRODUCTION}

The Aeon Patch is designed by its manufacturer as a unique and novel organic nanoscale patch that mildly stimulates a specific acupuncture point (CV6) to reduce "stress and inflammation." [For a general description of LifeWave patented nontransdermal patch technology, please refer to Nazeran et al., 2012]. Preliminary clinical research has shown that this Patch starts exerting a balancing effect on the autonomic nervous system (ANS) within as early as $10 \mathrm{~min}$ of its application as a consequence of eliciting a relaxation response (creating a calm and stress-free body state). Infrared thermal imaging has illustrated that this Patch reduces inflammation by lowering the C-reactive protein that can damage healthy tissue and even DNA [manufacturer's unpublished data].

Heart Rate Variability (HRV) signal refers to beat-to-beat variation of heart rate (HR) and represents the cyclical changes in HR. As HR is modulated by both parasympathetic and sympathetic inputs of the ANS, HRV can be utilized as an indirect and non-invasive marker of autonomic regulation and control under different physiological conditions (McMillan,

*Correspondence: Homer Nazeran

E-mail: hnazeran@utep.edu

Received July 10, 2014; Accepted October 29, 2014; Published

February 28, 2015

doi: http://dx.doi.org/10.5667/tang.2014.0024

(C)2015 by Association of Humanitas Medicine

This is an open access article under the CC BY-NC license.

(http://creativecommons.org/licenses/by-nc/3.0/)
2002). High HRV reflects an ANS that is adaptable and dynamically responsive to change whereas reduced HRV is indicative of an abnormal or restricted ability of the ANS in maintaining homeostasis (McMillan, 2002; Pumprla et al., 2002). Pharmacological studies and spectral analysis of the HRV signal have revealed two clear peaks in its power spectrum: a High Frequency (HF) and a Low Frequency (LF) component. The HF peak which is typically centered on 0.25 $\mathrm{Hz}(0.15-0.4 \mathrm{~Hz})$ arises from the coupling of the respiratory and cardiac cycle and is termed Respiratory Sinus Arrhythmia (RSA). Vagal blockade abolishes this HF peak suggesting that it is parasympathetically mediated. The LF peak, which is typically centered around $0.1 \mathrm{~Hz}(0.05-0.15)$, arises from blood pressure changes (Mayer-waves) and can be modified by both vagal and sympathetic blockade and therefore reflects both sympathetic and parasympathetic influences (Pumprla et al., 2002). The spectral band $0.003-0.05 \mathrm{~Hz}$, known as Very Low Frequency (VLF), is possibly related to the long-term regulatory mechanisms. A better measure of sympathetic modulation is the $\mathrm{LF} / \mathrm{HF}$, which is indicative of the balance between sympathetic and parasympathetic activity (sympathovagal balance) with a low frequency bias suggesting sympathetic dominance. The LF/HF is an index that relates the balance between sympathetic and parasympathetic parts of the autonomic nervous system and hence shows the level of sympathetic or parasympathetic dominance (stress or deep relaxation) in a subject.

A number of research studies in recent years have 
confirmed that higher variability in HR is indicative of a healthy ANS, while reduced variations in the HR is a sign of diseased or pathological state. Hundreds of research articles have been published on applications of HRV signal analysis. These articles have explored the relationships between HRV signal and blood pressure, myocardial infarction, nervous system, cardiac arrhythmia, diabetes, respiration, renal failure, gender, age, drugs, smoking, alcohol consumption, to name a few (Malik, 1996). HRV signal analysis seems to become even more popular in quantitative patient data analysis in the future. In short, HRV signal analysis provides a quantitative marker of the autonomic nervous system as the regulation mechanisms of beat-to-beat variations in the HR originate from the sympathetic and parasympathetic arms of the ANS.

Previous double-blind-placebo-controlled clinical studies in humans have shown that LifeWave patches produce a thermoregulating effect by mild stimulation of the acupuncture points on the body, promoting the parasympathetic nervous system activity. This parasympathetic dominance has a relaxing, stress reducing, or skin cooling effect (Nazeran, 2007; Nazeran et al., 2012). This in turn elicits targeted physiological responses.

To provide quantitative evidence and substantiate the claims that Aeon Patch reduces stress, it was necessary to acquire physiological data in a comfortable and nonintrusive fashion to assess and quantify the level of stress and its reduction by using well-proven and established analytical research tools in a double-blind-placebo-controlled investigation. Therefore, a small wearable wireless electrocardiographic (ECG) data acquisition system was used to capture ECG data from 50 volunteers under 3 different conditions: Control (C) - while wearing no Patch, Placebo (P) - while wearing a Placebo Patch for $20 \mathrm{~min}$, and Active (A) while wearing the Aeon Patch for $20 \mathrm{~min}$. After quality assurance and processing the ECG data, HRV signals were derived and analyzed to provide their spectral features (LF, HF, and $\mathrm{LF} / \mathrm{HF}$ ) among other parameters, which are quantitative measures of sympathetic and parasympathetic activities of the ANS. Statistical means were then deployed to test the hypothesis and evaluate the effects of the Aeon A Patch with reference to $\mathrm{C}$ and $\mathrm{P}$ conditions.

\section{MATERIALS AND METHODS}

The study protocol was reviewed and approved by the National Foundation for Energy Healing (project\#10-05-11-17). Participants were asked to keep well-hydrated and avoid taking any medications one day before data collection or drinking any stimulants (i.e. coffee) in the morning before participating in data collection. To assure adequate hydration, water bottles were provided to the subjects prior to the intervention and data collection. The study excluded subjects with cardiovascular disorders, those on 4 or more medications, those who were terminally ill, or had received chemotherapy in the last 12 months, those who had surgery in the previous 12 months, and those with HIV, and pregnant women. Both the investigator and the volunteers were blind to the A and P Patches as they looked exactly the same, were marked as Set 1 and Set 2 by the manufacturer (LifeWave LLC, San Diego, California, USA), and were assigned to subjects in a random fashion by the investigator through the research assistant responsible for data collection. So each subject could have been assigned to wear from either Set 1 or Set 2 first followed by wearing a patch from the other set next. ECG data collection involved using a CleveMed BioRadio 150 (Cleveland Medical Devices Inc,
Cleveland, OH, USA) device, and a Dell XPS laptop computer (Dell Inc, Austin, TX, USA). Three disposable $\mathrm{Ag} / \mathrm{AgCl}$ electrodes were applied to the right wrist (RA), left wrist (LA), and right ankle (RL as reference) after proper cleaning and preparation of the skin at the bioelectrode application sites.

After giving informed consent and filling out a demographic form, 50 well-hydrated volunteers [21 females and 29 males, age: $19-79(33.1 \pm 14.5)$ years, weight: 117 334 (177.5 \pm 51.5$)$ lbs, height: 5'-6',3" (5',7" $\pm 4 ")]$, participated in ECG data collection under 3 different conditions for a total duration of $15 \mathrm{~min}$ ( $5 \mathrm{~min} /$ condition). Condition1: C - while wearing no Patch, Condition 2: $\mathrm{P}$ or $\mathrm{A}$ - after wearing either the Placebo or the Aeon Patch for $20 \mathrm{~min}$, and Condition 3: A or P after wearing either the Aeon or the P Patch for 20 min. There was a washout period of 15 min between Conditions 2 and 3 . The investigator, research assistant, and volunteers were blind to the A and P Patches as they looked exactly the same, were marked as Set 1 and Set 2 by the manufacturer, and were assigned in a random fashion by the investigator. The subjects were instructed to apply the A or P Patch about 2 inches inferior to the navel (below belly button) on the CV6 acupuncture point (based upon instructions from the manufacture). Fig. 1. shows the anatomical position for applying the $\mathrm{P}$ or the A Patch. The hypothesis to be tested was: Wearing the Aeon Patch for $20 \mathrm{~min}$ reduces stress (elicits a deep relaxation response or produces a parasympathetic effect).

ECG data acquired (during 2011 and 2012) from the volunteers were quality assured offline and were recorded in a digital data base. These stored data were then processed to derive the HRV signals by deploying advanced digital signal processing/analysis algorithms, codes, and instructions developed at the Massachusetts Institute of Technology (MIT) and Harvard Medical School, which are available from PhysioNet or PhysioBank website [http://www.physionet.org /physiobank/]. To perform short-term HRV signal analysis, ECG signals from each volunteer were recorded for three $5 \mathrm{~min}$ intervals during each condition, while wearing no patch $\mathrm{C}$ or wearing either a $\mathrm{P}$ or an A Patch for $20 \mathrm{~min}$.

Power spectral analysis of HRV signals provides the basic information on how power within the signal is distributed as a function of frequency. Variations in the HR, occurring at the spectral frequency band of $0.15-0.4 \mathrm{~Hz}$, known as HF band, are indicative of parasympathetic (vagal) activity. Variations in the spectral band $0.05-0.15 \mathrm{~Hz}$, known as LF band, are linked to the sympathetic modulation, but this component includes

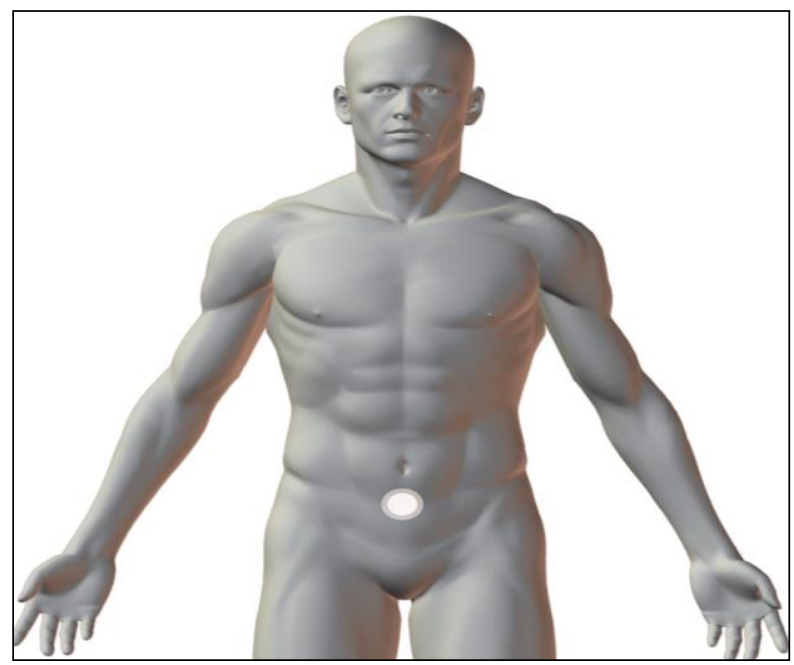

Fig. 1 Anatomical position for wearing the Placebo or the Y-Age Aeon Patch on CV6 acupuncture point 
Table 1a. HRV spectral analysis results for $C$ condition (healthy 27 years old female subject)

\begin{tabular}{ccccc}
\hline $\begin{array}{c}\text { Frequency Band } \\
\text { VLF }\end{array}$ & Peak (Hz) & Power $\left(\mathbf{m s}^{2}\right)$ & Power (\%) & $\begin{array}{c}\text { Power } \\
(\text { n.u. }) \%\end{array}$ \\
$(0-0.04 \mathrm{~Hz})$ & 0.0039 & 6039 & 35.1 & \\
$\begin{array}{c}\text { LF } \\
(0.04-0.15 \mathrm{~Hz})\end{array}$ & 0.0430 & 5690 & 33.1 & 51.0 \\
$\begin{array}{c}\text { HF } \\
(0.15-0.4 \mathrm{~Hz})\end{array}$ & 0.1523 & 5468 & 31.8 & 49.0 \\
\hline $\begin{array}{c}\text { Total } \\
\text { LF/HF }\end{array}$ & 17197 & & \\
\hline
\end{tabular}

Table 1b. HRV spectral analysis results for $P$ condition (healthy 27 years old female subject)

\begin{tabular}{ccccc}
\hline $\begin{array}{c}\text { Frequency Band } \\
\text { VLF }\end{array}$ & Peak $(\mathbf{H z})$ & Power $\left(\mathbf{m s}^{2}\right)$ & Power $(\%)$ & $\begin{array}{c}\text { Power } \\
(\mathbf{n . u .}) \%\end{array}$ \\
\hline $\begin{array}{c}(0-0.04 \mathrm{~Hz}) \\
\text { LF }\end{array}$ & 0.0039 & 51074 & 69.4 & \\
$(0.04-0.15 \mathrm{~Hz})$ & 0.0430 & 16263 & 22.1 & 72.1 \\
$\begin{array}{c}\text { HF } \\
(0.15-0.4 \mathrm{~Hz})\end{array}$ & 0.1523 & 6285 & 8.5 & 27.9 \\
\hline Total & & 73622 & & \\
\hline LF/HF & & 2.588 & \\
\hline
\end{tabular}

Table 1c. HRV spectral analysis results for $A$ condition (healthy 27 years old female subject)

\begin{tabular}{ccccc}
\hline Frequency Band & Peak (Hz) & Power $\left(\mathbf{m s}^{2}\right)$ & Power (\%) & $\begin{array}{c}\text { Power } \\
\text { (n.u.) \% }\end{array}$ \\
$\begin{array}{c}\text { VLF } \\
(0-0.04 \mathrm{~Hz})\end{array}$ & 0.0039 & 16167 & 47.7 & \\
$\begin{array}{c}\text { LF } \\
(0.04-0.15 \mathrm{~Hz})\end{array}$ & 0.0430 & 7406 & 36.5 & 69.8 \\
$\begin{array}{c}\text { HF } \\
(0.15-0.4 \mathrm{~Hz})\end{array}$ & 0.1680 & 6625 & 15.8 & 30.2 \\
\hline $\begin{array}{c}\text { Total } \\
\text { LF/HF }\end{array}$ & 30198 & & \\
\hline
\end{tabular}

some parasympathetic influence (sympathovagal influences). The spectral band $0.003-0.05 \mathrm{~Hz}$, known as VLF, is possibly related to the long-term regulatory mechanisms. It is well established that the level of physical activity is clearly reflected in the HRV power spectrum. For example, when a healthy subject stands up, there is an increase of HRV in the $L F$ spectral band, which is considered to be an estimate of the sympathetic influence on the heart. Consequently, the $L F / H F$ is a quantitative indication of sympathovagal balance (Pumprla et al., 2002).

The spectral components, namely the VLF, LF, HF and LF/HF of the HRV signals, as well as a number of HRV timedomain, frequency-domain and nonlinear dynamics measures were calculated from 5-min ECG recordings under 3 different conditions while wearing no patch or wearing the $\mathrm{P}$ or A Patches for $20 \mathrm{~min}$. Kubios HRV - Heart Rate Variability Software Analysis Software developed by the Biosignal and Medical Imaging Group at the University of Eastern Finland was used to calculate the time-domain, frequency-domain (spectral), and nonlinear dynamics features of the HRV signals [http://kubios.uku.fi/]. The power spectral components of the HRV signals were determined in normalized units (n.u.), which represent the relative values of each power component in proportion to the total power minus the $V L F$ component. The representation of $L F$ and $H F$ in n.u. emphasizes the controlled and balanced behavior of the two branches of the ANS (McMillan, 2002). It has been shown that $L F$ and $H F$ can increase under different physical and physiological conditions. An increase in normalized LF is observed during $90^{\circ}$ tilt, standing, mental stress and moderate exercise in healthy subjects. Conversely, an increase in normalized HF is induced by controlled respiration, cold stimulation of the face and rotational stimuli (Malik, 1996).

\section{RESULTS}

Tables $1 \mathrm{a}, 1 \mathrm{~b}$, and $1 \mathrm{c}$ show the results of frequency-domain analysis of 5 min long datasets acquired from a healthy female subject (Age: 27 years old, Weight: $150 \mathrm{lb}$, Height: 5', 8") under 3 different conditions: baseline or $\mathrm{C}$, wearing the $\mathrm{P}$ Patch for 20 min, and wearing the A Patch for 20 min, respectively as an example. Comprehensive HRV analysis results and graphs for this female subject under these conditions as produced by the

Table 2a. HRV spectral analysis results for $C$ condition (healthy 25 years old male subject)

\begin{tabular}{ccccc}
\hline $\begin{array}{c}\text { Frequency Band } \\
\text { VLF }\end{array}$ & Peak $(\mathbf{H z})$ & Power $\left(\mathbf{m s}^{2}\right)$ & Power $(\%)$ & $\begin{array}{c}\text { Power } \\
(\text { n.u. }) \%\end{array}$ \\
$(0-0.04 \mathrm{~Hz})$ & 0.0391 & 1318 & 17.3 & \\
$\begin{array}{c}\text { LF } \\
(0.04-0.15 \mathrm{~Hz})\end{array}$ & 0.1133 & 4498 & 59.1 & 71.5 \\
$\begin{array}{c}\text { HF } \\
(0.15-0.4 \mathrm{~Hz})\end{array}$ & 0.1523 & 1797 & 23.6 & 28.5 \\
\hline $\begin{array}{c}\text { Total } \\
\text { LF/HF }\end{array}$ & 7613 & & \\
\hline
\end{tabular}

Table 2b. HRV spectral analysis results for $P$ condition (healthy 25 years old male subject)

\begin{tabular}{ccccc}
\hline $\begin{array}{c}\text { Frequency Band } \\
\text { VLF }\end{array}$ & Peak $(\mathbf{H z})$ & Power $\left(\mathbf{m s}^{2}\right)$ & Power $(\%)$ & $\begin{array}{c}\text { Power } \\
(\text { n.u. }) \%\end{array}$ \\
$(0-0.04 \mathrm{~Hz})$ & 0.0039 & 3404 & 50.0 & \\
$\begin{array}{c}\text { LF } \\
(0.04-0.15 \mathrm{~Hz})\end{array}$ & 0.0430 & 2616 & 38.4 & 76.9 \\
$\begin{array}{c}\text { HF } \\
(0.15-0.4 \mathrm{~Hz})\end{array}$ & 0.1523 & 786 & 11.5 & 23.1 \\
$\begin{array}{c}\text { Total } \\
\text { LF/HF }\end{array}$ & 6806 & & \\
\hline
\end{tabular}

Table 2c. HRV spectral analysis results for $A$ condition (healthy 25 years old male subject)

\begin{tabular}{ccccc}
\hline $\begin{array}{c}\text { Frequency Band } \\
\text { VLF }\end{array}$ & Peak $(\mathrm{Hz})$ & Power $\left(\mathbf{m s}^{2}\right)$ & Power (\%) & $\begin{array}{c}\text { Power } \\
(\text { n.u. }) \%\end{array}$ \\
$(0-0.04 \mathrm{~Hz})$ & 0.0039 & 4469 & 47.7 & \\
$\begin{array}{c}\text { LF } \\
(0.04-0.15 \mathrm{~Hz})\end{array}$ & 0.0430 & 3423 & 36.5 & 69.8 \\
$\begin{array}{c}\text { HF } \\
(0.15-0.4 \mathrm{~Hz})\end{array}$ & 0.1523 & 1481 & 15.8 & 30.2 \\
\hline $\begin{array}{c}\text { Total } \\
\text { LF/HF }\end{array}$ & & 9374 & & \\
\hline
\end{tabular}


Table 3. Statistical measures for $\mathrm{LF} / \mathrm{HF}$ for $\mathrm{P}$ and $\mathrm{A}$ conditions and $\%$ reduction of LF/HF Active: Placebo $(n=30)$.

\begin{tabular}{cccc}
\hline $\begin{array}{c}\text { Statistical } \\
\text { Measure }\end{array}$ & $\begin{array}{c}\text { LF/HF Placebo } \\
\text { Patch }\end{array}$ & $\begin{array}{c}\text { LF/HF Aeon } \\
\text { Patch }\end{array}$ & $\begin{array}{c}\text { \% reduction in } \\
\text { LF/HF } \\
\text { compared to } \\
\text { Placebo }\end{array}$ \\
\hline $\begin{array}{c}\text { Mean } \\
\text { Standard } \\
\text { Deviation }\end{array}$ & 1.960 & 1.500 & 24 \\
\hline $\boldsymbol{p}$-value $=\mathbf{0 . 0 4 7}$ & 1.100 & 1.000 & 9 \\
\hline
\end{tabular}

Kubios-HRV Analysis Software are available electronically from the author upon making an email request.

Tables $2 \mathrm{a}, 2 \mathrm{~b}$, and $2 \mathrm{c}$ show the results of frequency-domain analysis of $5 \mathrm{~min}$ long datasets acquired from a healthy male subject (Age: 25 years old, Weight: $250 \mathrm{lb}$, Height: 5', 10") under 3 different conditions: baseline or $\mathrm{C}$, wearing the $\mathrm{P}$ Patch for $20 \mathrm{~min}$, and wearing the A Patch for $20 \mathrm{~min}$, respectively as a second example. Comprehensive HRV analysis results and graphs for this male subject under these conditions are available electronically from the author upon making an email request.

Spectral features of the derived HRV signals were used to assess the parasympathetic effect or the deep relaxation response elicited by the Y-Age Aeon Patch for the study population. Data from the 3 conditions: $\mathrm{C}, \mathrm{A}$, and $\mathrm{P}$ were first quality assured after data collection and those subjects who achieved a parasympathetic response after wearing the Active patches within $20 \mathrm{~min}$ were identified as responders. Out of the 50 subjects, $30(60 \%)$ could achieve a relaxed state after wearing the Aeon Patch as early as $20 \mathrm{~min}$. Table 3 shows the statistical summary of the LF/HF for the responders under $\mathrm{P}$ and A conditions. The \% reductions in $\mathrm{LF} / \mathrm{HF}$ ranged from $2 \%$ to $56 \%$ in responders.

Statistical analysis (one-sample inference) was used to compare the LF/HF of the responders under these 3 different conditions. The normalized LF/HF indicative of sympathovagal balance decreased significantly $(24 \% \pm 9 \%$ after $20 \mathrm{~min})$ in condition A compared to condition $\mathrm{P}$ with a $p$-value $<0.047$ (n $=30$ ) in responders. There was also a statistically significant difference (\% reduction in LF/HF due to wearing the Aeon Patch compared to when no patch was worn) between LF/HF spectral features between conditions $\mathrm{A}$ and $\mathrm{C}$ with a $p$-value < 0.05 . There was also a nearly statistically significant difference between spectral features in condition P to condition C. Such differences in spectral parameters are indicative of the Placebo Effect. This is an indication of how the subjects responded to the feeling of wearing a patch.

To evaluate the effectiveness of the Aeon Patch in eliciting a deep relaxation response with time, a single subject pilot investigation was carried out (in early 2011) before initiation of the double-blind-placebo-controlled (DBPC) study. This was a critical preliminary step to inform the data collection and study protocol design in the DBPC. The results of the pilot investigation enabled the principal investigator to arrive at a reasonable time estimate (about $20 \mathrm{~min}$ ) to elicit a relaxation response in the majority of population by using the Aeon Patch. This time factor was very important in the data collection protocol as it was essential to limit the amount of time commitment from each subject (to about $1 \mathrm{~h}$ ) for ECG data collection and consequently reduce the subject inconvenience. Tables $4 \mathrm{a}, 4 \mathrm{~b}$, and $4 \mathrm{c}$ show the results of frequency-domain analysis of 5 min long datasets acquired from a healthy male subject (age: 28 years old, weight: $175 \mathrm{lb}$, height: 6') at 3 different times: baseline or $\mathrm{C}$, after wearing the Aeon Patch for
$1 h$ and after wearing the Aeon Patch for $2 h$. Comprehensive HRV analysis results and graphs for this male subject under these conditions as produced by the Kubios-HRV Analysis Software are available electronically from the author upon making an email request.

\section{DISCUSSION}

From the spectral analysis of the HRV signals of $5 \mathrm{~min}$ recordings of ECG datasets acquired in this study population and the statistical analysis of the results for 50 subjects, the following observations could be made:

1. There was a nearly significant difference $(p=0.071)$ between spectral parameters indicative of the relaxation response or stress reduction effect (as measured by normalized LF/HF power) when the subjects wore the $\mathrm{P}$ Patch compared with when the subjects wore no Patch (C condition). Such differences (a reduction of $25 \% \pm 60 \%$ ) in spectral parameters $(\mathrm{LF} / \mathrm{HF})$ are indicative of the $\mathrm{P}$ Effect.

Table 4a. HRV spectral analysis results for $C$ condition (healthy 28 years old male subject)

\begin{tabular}{ccccc}
$\begin{array}{c}\text { Frequency Band } \\
\text { VLF }\end{array}$ & Peak $(\mathbf{H z})$ & Power $\left(\mathbf{m s}^{2}\right)$ & Power $(\%)$ & $\begin{array}{c}\text { Power } \\
(\mathbf{n . u .}) \%\end{array}$ \\
$\begin{array}{c}(0-0.04 \mathrm{~Hz}) \\
\begin{array}{c}\text { LF } \\
(0.04-0.15 \mathrm{~Hz})\end{array}\end{array}$ & 0.0430 & 2502 & 32.5 & 84.2 \\
$\begin{array}{c}\text { HF } \\
(0.15-0.4 \mathrm{~Hz})\end{array}$ & 0.1523 & 471 & 6.1 & 15.8 \\
\hline Total & & 7709 & & \\
\hline LF/HF & 5.317 & & \\
\hline
\end{tabular}

Table 4b. HRV spectral analysis results after wearing the Aeon Patch for $1 \mathrm{~h}$ (healthy 28 years old male subject)

\begin{tabular}{|c|c|c|c|c|}
\hline Frequency Band & Peak (Hz) & Power $\left(\mathrm{ms}^{2}\right)$ & Power (\%) & $\begin{array}{r}\text { Power } \\
\text { (n.u.) \% }\end{array}$ \\
\hline $\begin{array}{c}\text { VLF } \\
(0-0.04 \mathrm{~Hz})\end{array}$ & 0.0039 & 10061 & 76.2 & \\
\hline $\begin{array}{c}\mathrm{LF} \\
(0.04-0.15 \mathrm{~Hz})\end{array}$ & 0.0430 & 2112 & 16.0 & 67.2 \\
\hline $\begin{array}{c}\mathrm{HF} \\
(0.15-0.4 \mathrm{~Hz})\end{array}$ & 0.2813 & 1031 & 7.8 & 32.8 \\
\hline Total & & 13205 & & \\
\hline $\mathrm{LF} / \mathrm{HF}$ & & 2.049 & & \\
\hline
\end{tabular}

Table 4c. HRV spectral analysis results after wearing the Aeon Patch for $2 \mathrm{~h}$ (healthy 28 years old male subject)

\begin{tabular}{ccccc}
\hline $\begin{array}{c}\text { Frequency Band } \\
\text { VLF }\end{array}$ & Peak $(\mathbf{H z})$ & Power $\left(\mathbf{m s}^{2}\right)$ & Power (\%) & $\begin{array}{c}\text { Power } \\
(\mathbf{n . u .}) \%\end{array}$ \\
$\begin{array}{c}(0-0.04 \mathrm{~Hz}) \\
\text { LF }\end{array}$ & 0.0039 & 878 & 60.4 & \\
$(0.04-0.15 \mathrm{~Hz})$ & 0.0430 & 300 & 20.6 & 52.1 \\
$\begin{array}{c}\text { HF } \\
(0.15-0.4 \mathrm{~Hz})\end{array}$ & 0.3164 & 276 & 19.0 & 47.9 \\
\hline Total & 1454 & & \\
\hline LF/HF & 1.087 & & \\
\hline
\end{tabular}


This is an indication of how the subjects responded to the feeling of wearing a patch.

2. After wearing the Aeon Patch, the majority of the participants $(60 \%)$ called the responders could achieve stress relief within as early as $20 \mathrm{~min}$. The degree of stress relief or relaxation response in responders due to wearing the Aeon Patch ranged from $2 \%$ to $56 \%$. This is indicative of the fact that different individuals relax at different rates under the influence of these patches.

3. There was a consistent decrease in the normalized \% of LF Power (indicative of the Sympathetic dominance) when the subjects wore the Aeon Patch compared with the condition when the subjects wore the P Patch. In the female subject (Table 1), LF Power (n.u.)\% decreased from $72.1 \%$ to $69.8 \%$. For the male subject (Table 2), LF Power (n.u.)\% decreased from $76.9 \%$ to $69.8 \%$. There was also a consistent decrease in LF Power (n.u.)\% with time (Table 4). This value decreased from $84.2 \%$ to $67.2 \%$ after $1 \mathrm{~h}$ with a further reduction to $52.1 \%$ after $2 \mathrm{~h}$, due to wearing the Aeon Patch compared to condition $\mathrm{C}$.

4. There was an increase in the normalized $\%$ of $H F$ Power (indicative of the Parasympathetic dominance) when the subjects wore the Aeon Patch compared with the condition when the subjects wore the P Patch. In the female subject (Table 1), HF Power (n.u.)\% increased from $27.9 \%$ to $30.2 \%$. For the male subject (Table 2), HF Power (n.u.)\% increased from $23.1 \%$ to $30.2 \%$. There was also a consistent increase of HF Power (n.u.)\% with time (Table 4). This value increased from $15.8 \%$ to $32.8 \%$ after $1 \mathrm{~h}$ with a further elevation to $47.9 \%$ after $2 \mathrm{~h}$, due to wearing the Aeon Patch.

5 . There was a statistically significant $(p<0.047)$ decrease in the normalized $L F / H F$ power when the subjects wore the Aeon Patch for 20 min compared with the condition when the subjects wore the P Patch for $20 \mathrm{~min}$.

6 . There was a large decrease in the normalized $L F / H F$ power when the subject wore the Aeon Patch for $1 h$ compared with the baseline (C) condition. The effectiveness of the Aeon Patch in eliciting a deeper relaxation response with time was clearly demonstrated in the single subject investigation that caused a reduction of normalized LF/HF power from 5.317 at baseline to 2.049 (more than 2.5 times reduction) after $1 \mathrm{~h}$ and to 1.087 (with a further two-fold decrease) after $2 \mathrm{~h}$.

7. A statistical comparison was performed between the average male and female LF/HF values in all the responders. No statistically significant differences $(p=0.7)$ were found between these two groups.

Based on these observations it could be concluded that wearing the Aeon Patch for 20 min elicited an enhanced parasympathetic response and could enable the majority of the participants to achieve a reduced stress state with varying degrees. This response could be quantified by a reduction in normalized LF/HF power. The single subject investigation revealed that the effectiveness of Aeon Patch in causing a stress relief increases with time. The statistical results revealed that the Aeon Patch showed a significant effect $(p<0.047)$ compared to the Placebo Patch in reducing the normalized $\mathrm{LF} / \mathrm{HF}$ power after $20 \mathrm{~min}$, there was a large reduction in LF/HF with time. The data also showed the Placebo Effect, which is an indication of how the subjects responded to the feeling of wearing a patch. Based upon these results, it is reasonable to expect that wearing the Aeon Patch may cause a significant relaxation effect after $30 \mathrm{~min}$ in the majority of the population.

\section{ACKNOWLEDGEMENTS}

The author would like to thank Mr Carlos Ramos for his contributions to subject recruitment, high quality ECG data collection, data quality assurance, and signal processing. The author also acknowledges LifeWave LLC for providing the patches for this investigation and a small grant to cover data collection, research assistance, subject recruitment, subject incentives and transportation costs to and from the study site.

\section{CONFLICT OF INTEREST}

The author does not have any conflict of interest in the present study.

\section{REFERENCES}

Malik M. Task Force of the European Society of Cardiology the North American Society of Pacing Electrophysiology. Heart rate variability. Standards of measurement, physiological interpretation, and clinical use. Circulation. 1996;93:1043-1065.

McMillan DE. Interpreting heart rate variability sleep/wake patterns in cardiac patients. J Cardiovas Nurs 2002;17:69-81.

Nazeran H. Heart Rate Variability Signal Parameters Quantify Skin cooling Effect of Energy Patches During Rest and Exercise in Young Healthy Individuals. In Biomedical Engineering Recent Developments. Otto Wilson, Binh Tran, Jafar Vossoughi ed. (Maryland, USA; medical and engineering publishers, inc). pp. 13-19, 2007.

Nazeran H, Blake-Greenberg S, Haltiwanger E. Mild stimulation of stomach 36 acupuncture point by organic nanoscale SP6 patch improves cellular physiologic functional status of different organs. TANG. 2012;2:e10.

Pumprla J, Howorka K, Groves D, Chester M, Nolan J. Functional assessment of heart rate variability: physiological basis and practical applications. Int J Cardiol. 2002:84:1-14. 